\title{
Hormonal control of cardiac regenerative potential
}

\author{
Alexander V Amram ${ }^{1,2}$, Stephen Cutie ${ }^{1,2}$ and Guo N Huang (1) 1,2 \\ ${ }^{1}$ Department of Physiology, Cardiovascular Research Institute, University of California, San Francisco, San Francisco, California, USA \\ ${ }^{2}$ Eli and Edythe Broad Center for Regeneration Medicine and Stem Cell Research, University of California, San Francisco, San Francisco, California, USA
}

Correspondence should be addressed to G N Huang: Guo.Huang@ucsf.edu

\begin{abstract}
Research conducted across phylogeny on cardiac regenerative responses following heart injury implicates endocrine signaling as a pivotal regulator of both cardiomyocyte proliferation and heart regeneration. Three prominently studied endocrine factors are thyroid hormone, vitamin $\mathrm{D}$, and glucocorticoids, which canonically regulate gene expression through their respective nuclear receptors thyroid hormone receptor, vitamin $D$ receptor, and glucocorticoid receptor. The main animal model systems of interest include humans, mice, and zebrafish, which vary in cardiac regenerative responses possibly due to the differential onsets and intensities of endocrine signaling levels throughout their embryonic to postnatal organismal development. Zebrafish and lower vertebrates tend to retain robust cardiac regenerative capacity into adulthood while mice and other higher vertebrates experience greatly diminished cardiac regenerative potential in their initial postnatal period that is sustained throughout adulthood. Here, we review recent progress in understanding how these three endocrine signaling pathways regulate cardiomyocyte proliferation and heart regeneration with a particular focus on the controversial findings that may arise from different assays, cellular-context, age, and species. Further investigating the role of each endocrine nuclear receptor in cardiac regeneration from an evolutionary perspective enables comparative studies between species in hopes of extrapolating the findings to novel therapies for human cardiovascular disease.
\end{abstract}

\author{
Key Words \\ - heart regeneration \\ - cardiomyocyte \\ proliferation \\ - thyroid hormones \\ - vitamin D \\ - glucocorticoids
}

\section{Introduction}

Cardiovascular disease is the leading cause of mortality worldwide (1), posing an unprecedented need to investigate the underlying mechanisms of heart regeneration across phylogeny to develop novel therapies (2). A prominent theory for the phylogenetic divergence in cardiac regenerative capacity is the species-dependent onset and intensity of endocrine signaling throughout development (2). The ability for vertebrates to regenerate damaged heart tissue by replacing lost cardiomyocytes (CMs) varies dramatically $(3,4)$. Higher vertebrates such as humans and mice display minimal regenerative capacity following cardiac damage in adulthood $(2,5)$, while lower vertebrates such as zebrafish can regenerate lost heart muscle fully within weeks and into adulthood $(4,6)$. Even embryonic and neonatal higher vertebrates have been found to retain robust cardiac regenerative potential (5). Compelling genetic experiments in newborn mice and adult zebrafish regeneration have revealed that new CMs originate from pre-existing CMs through rapid proliferation $(5,6)$. However, most mammals undergo a postnatal rise in $\mathrm{CM}$ multinucleation and polyploidization and concurrent $\mathrm{CM}$ cell-cycle arrest (5), leading scientists to investigate the underlying causes of postnatal decreases in cardiac regenerative potential in higher vertebrates with a prominent theory being the corresponding alteration of endocrine signaling (2).
Endocrine Connections (2021) 10, R25-R35

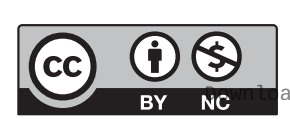

This work is licensed under a Creative Commons Attribution-NonCommercial 4.0 International License. ded from Bioscientifica.com at 04/26/2023 09:48:59AM 
Recent findings suggest that the suppression of $\mathrm{CM}$ proliferation and subsequent restriction of cardiac regeneration post-birth coincides with the augmentation of metabolic rates, thermogenesis, and endocrine signaling across species (2). The argument that evolutionarily conserved mechanisms contribute to cardiac regenerative capacity forms the basis for comparative studies between differentspecies in hopes to extrapolate the findings to novel therapies for human cardiovascular disease (4). Certain endocrine factors have recently been investigated for their contributions to the evolutionarily divergent potentials of $\mathrm{CM}$ proliferation and heart regeneration, including thyroid hormone, vitamin D, and glucocorticoids. A recent phylogenetic analysis of CM ploidy, a proxy of cardiac regenerative potential, across 41 vertebrate species suggests that the evolutionary transition from ectothermy to endothermy, primarily driven by thyroid hormone, provides a compelling evolutionary explanation for the divergent cardiac regenerative capacity across species (2). The study highlights the significant regulatory role of endocrine signaling in cardiac regeneration and provides grounds for studying the potential cardiac regulatory roles of other hormones. As of recent, studies performed across species suggest that thyroid hormone, vitamin D, and glucocorticoids may exert influences on cardiac regeneration with their variability arising from assay, cell, developmental, and species-dependent differences (Fig. 1).

\section{Roles of thyroid hormones in regulating cardiomyocyte proliferation and cardiac regeneration}

Thyroid hormones are produced by thyroid glands and bind their cognate receptor, thyroid hormone receptor

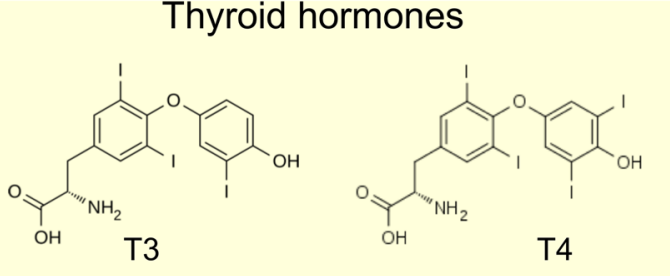

TR
Vitamin D

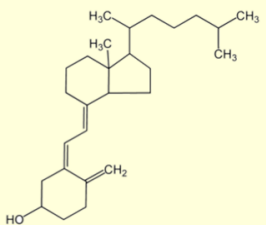

VDR
Glucocorticoid

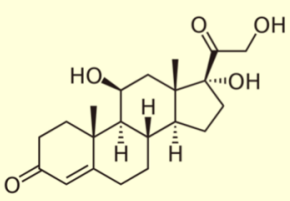

GR

\section{Species, age, dose, duration, assay-dependent}

\section{Cardiomyocyte proliferation}

\section{Heart regeneration}

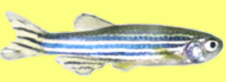

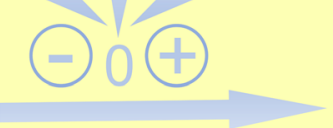
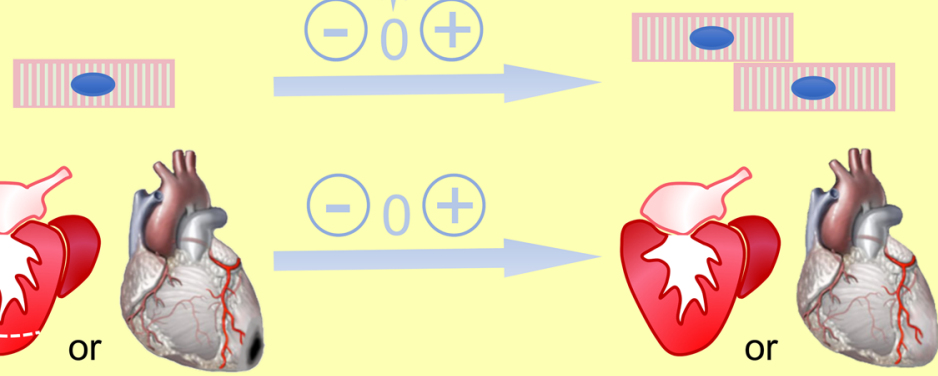

or
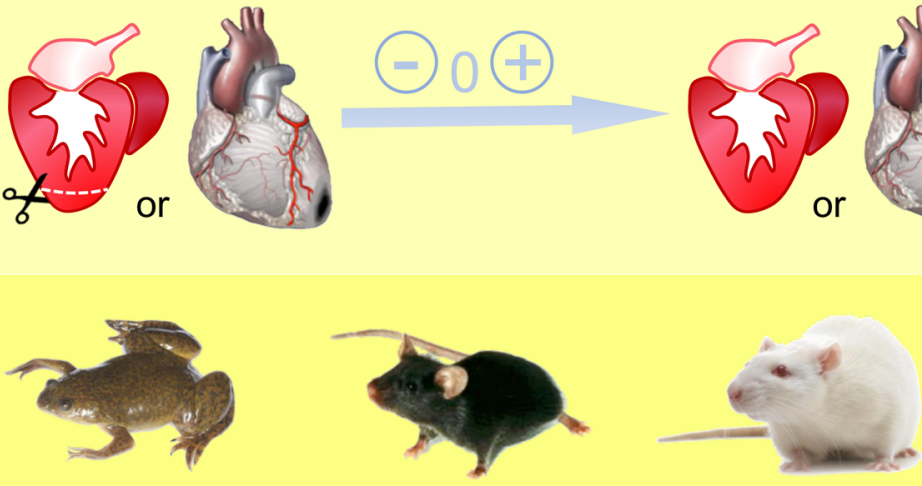

Figure 1

Thyroid hormones, vitamin $\mathrm{D}$, and glucocorticoids have been reported to execute positive, neutral or negative influences on cardiomyocyte proliferation and heart regeneration which could be species, age, dose, duration and assay dependent.

https://ec.bioscientifica.com

https://doi.org/10.1530/EC-20-0503 (c) 2021 The authors Published by Bioscientifica Ltd

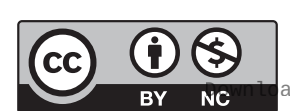

This work is licensed under a Creative Commons Attribution-NonCommercial 4.0 International License. ded from Bioscientifica.com at 04/26/2023 09:48:59AM 
(TR), for downstream gene activation and effector regulation (7). In higher vertebrates, triiodothyronine (T3) and thyroxine (T4) are the two most prominent forms of thyroid hormone (7). Thyroid gland follicular cells synthesize and secrete T4 and T3 at a roughly 80:20 ratio (7). Through the circulatory system, T4 and T3 are transported to target cells (7). Most T4 is converted locally to T3 by deiodinase enzymatic activity (7). Thyroid hormones regulate the function of target cells mainly through TR, a member of the nuclear receptor superfamily (7). TR predominantly manifests in the isoforms TR $\alpha$ and $\operatorname{TR} \beta$ (7). Upon ligand binding, these receptors turn on target gene expression as their canonical pathway (7). In recent years, non-genomic functions of thyroid hormone receptors in regulating channels and enzymatic activity have been reported as the non-canonical pathway, including specific cardiometabolic contributions (8). For instance, Hönes et al. observed that despite the loss of canonical $\mathrm{TH}$ binding to its nuclear receptors, the physiological effects of TH on heart rate, thermogenesis, cholesterol and serum triglycerides, and blood glucose were largely preserved via non-canonical $\mathrm{TH}$ signaling (9). Canonical TH signaling was still found to primarily regulate the hypothalamic-pituitary-thyroid axis - as TH binding to $\operatorname{TR} \beta$ was required for inhibition of thyroidstimulating hormone - and skeletal development - as inability of $\mathrm{TH}$ to bind $\mathrm{TR} \alpha$ resulted in the same stunted skeletal development as loss of TR $\alpha$ (9).

The physiological effect of thyroid hormone on $\mathrm{CM}$ proliferation is controversial. Thyroid hormone has been reported to either inhibit (10), enhance (11), or have no effect (12) on CM proliferation. However, these conclusions relied on either primary CM culture in vitro or fetal thyroidectomy experiments, which can be profoundly influenced by cell conditions, medium compositions, and surgery complications $(10,11,12)$. Several studies in neonatal rodents support that thyroid hormones promote CM proliferation in vivo (13). Naqvi et al. reported a new wave of CM division in preadolescent mice and a primary role of T3 in stimulating this process (13). Notably, they also observed that the acute inhibition of thyroid hormone activity via propylthiouracil (PTU) treatment between P7 and P14 blunted the CM proliferative burst they observed at P15 (13). Although two other groups disputed any CM expansion in this time window with compelling evidence, including design-based stereology that does not depend on CM isolation efficiency, the role of thyroid hormone signaling was not addressed in these later reports $(14,15)$. Recently, T3 treatment of neonatal mice at P2 and P3 (20 ng per animal) was shown to increase CM proliferation as supported by increases in EdU incorporation from $0.01 \%$ to greater than 7\%, mitotic CMs by approximately seven folds, and the total CM number by approximately 10\% (16).

In contrast, there are also compelling pharmacological and genetic experiments arguing that postnatal thyroid hormones inhibit CM proliferation in vivo $(2,17)$. An earlier study of hyperthyroidism in neonatal rats showed daily T4 administration (5-20 mg per g body weight) impeded hyperplastic myocyte growth and promoted hypertrophic growth even after exogenous T4 cessation with sustained significant increases in myocyte length (17). During the 12-day exogenous T4 treatment period, the corresponding rats experienced a 31\% increase in cell volume, a 30\% increase in their heart weight to body weight ratio, and a 19\% decrease in the mean number of ventricular myocytes as compared to controls, indicating exogenous T4 promotes hypertrophic growth and inhibits CM proliferation in neonatal rats (17). Conversely, Hirose et al. recently observed that both pharmacological and genetic inactivation of thyroid hormone signaling delays neonatal CM cell-cycle exit in a cell-autonomous manner, supporting the physiological role of thyroid hormones in suppressing CM cell cycle activity (2). In Hirose et al.'s study, the pharmacological treatment of mouse CMs with PTU, an inhibitor of thyroid hormone synthesis, produced a three-fold increase in proliferating diploid CMs (2). Notably, PTU was administered for a longer window than in Naqvi et al. from E13.5 through P14 (2), instead of starting at P7 (13). Furthermore, genetic engineering of mutant mice to express CM-specific dominant negative (DN) TR $\alpha$ yielded an approximately 2.3-fold increase in CM numbers and a 3.5-fold increase in diploid proliferating CMs (2).

While further work to reproduce the previous findings and elucidate the apparently disparate observations of the effects of thyroid hormone activity on CM proliferation is called for, the observed discrepancies between these studies may be partially explained by different doses of exogenous thyroid hormones and inhibitors such as PTU, and durations of treatments used in these studies $(2,10,11,12,18)$. It is well known that the effects of thyroid hormones are dependent on dose and duration. At high doses (5 mg per $100 \mathrm{~g}$ body weight T3) with extended duration, thyroid hormone administration in adult rats induces hypermetabolism and body weight loss (18), which may affect heart development and function indirectly when given to neonatal rodents. Conversely, short-term inhibition of thyroid hormone synthesis or exogenous thyroid hormone administration may lead to negative feedback mechanisms that could perturb the

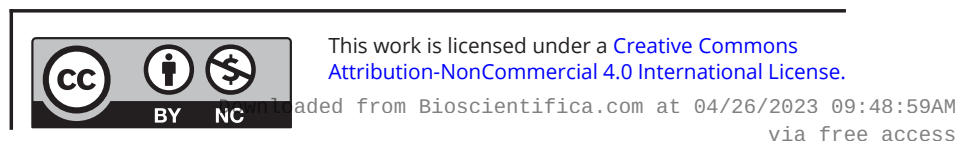


regulatory network after drug withdrawal. Thus, cautions should be taken to interpret these results. It also remains possible that different levels of sub- and supraphysiological thyroid hormone activity may not have identical effects on CM proliferation. In addition, different protein marks used to identify CMs and different methods of CM number quantification (stereology vs enzymatic digestion) may contribute to different observations. Myocyte-specific perinuclear formation of pericentriolar marker 1 (PCM1) (2) allows for the identification of CM nuclei, while cardiac troponin T (cTnT) reliably labels CM cell bodies (19). PCM-1, while expressed in most cell types, only localizes to a perinuclear shell or ring around CM and skeletal muscle nuclei $(20,21)$, and thus, allows for specific identification of CM nuclei rather than CM cell bodies (19). Because increases of $\mathrm{CM}$ cell-cycle activity occur during both hyperplastic and hypertrophic responses, accurate quantification of total $\mathrm{CM}$ cell number is essential to distinguish true proliferation from hypertrophic growth. Design-based stereological analysis is one such method for quantifying total $\mathrm{CM}$ cell number, although studies that used stereology to assess total CM number during human development have reported different observations on whether substantial CM expansion occurs leading up to adulthood $(19,22)$. Whether stereology or enzymatic isolation is a more accurate method for quantifying total $\mathrm{CM}$ number is a relevant question that future research should help answer.

Consistent with thyroid hormone's modulation of $\mathrm{CM}$ proliferation, thyroid hormones function as essential regulators of cardiac regenerative potential. Hirose et al. demonstrated that the inactivation of thyroid hormone signaling in adult mice endows them with cardiac regenerative potential (2). Mice with a CM-specific TR $\alpha$ DN mutation exhibit an approximately $62 \%$ decrease in fibrotic scar tissue as compared to controls 1 month following ischemia-reperfusion injury (2). Conversely, exogenous thyroid hormone administration (5 nM) inhibits zebrafish heart regeneration (2). Although adult zebrafish normally retain the ability to fully regenerate damaged heart tissue, exogenous T3 impairs cardiac regenerative capacity with sustained prominent scarring in injured areas (2). Altogether, these results demonstrate that thyroid hormones are essential regulators of cardiac regeneration across phylogeny.

Thyroid hormones also promote CM differentiation and maturation. They have been widely used to induce maturation of CMs derived from mouse embryonic stem cells and human inducible pluripotent stem cells $(23,24)$. Through RNA-seq and chromatin immunoprecipitation- seq analyses of genetically engineered mice, Hirose et al. identified many direct thyroid hormone receptor target genes functioning in mitochondrial biogenesis, myofibrillogenesis, calcium-handling protein expression, and electrophysiological changes (2). One of these targets is carnitine palmitoyltransferase 2 (Cpt2) (2), which encodes an enzyme essential for beta oxidation of longchain fatty acids in the mitochondria. Hirose et al. found that Cpt2 expression was reduced to $60 \%$ in thyroid hormone receptor mutant hearts, and Cpt2 heterozygous mutant mice resulted in enhanced CM proliferation, although the phenotype was mild compared with mice with thyroid hormone receptor inactivation (2). It is plausible that other thyroid hormone downstream genes are also crucial in driving CM cell-cycle withdrawal, while promoting CM differentiation and maturation.

Cardiac regenerative studies with thyroid hormone mainly focus on CM-specific regeneration, but growing research indicates that thyroid hormone also modulates regeneration through non-cell-autonomous mechanisms such as interstitial proteins (25) and the extracellular matrix (ECM) (26). The collagen matrix is an essential component of myocardial structural and functional integrity and originates from cardiac fibroblasts (25). Increasing cardiac fibrosis is characteristic of the aging process in mammals (25), coincides with postnatal increases in thyroid hormone circulation, and corresponds with the subsequent transition from hyperplastic to hypertrophic heart growth (27). A study conducted on rats demonstrated that upon the administration of exogenous L-thyroxine (100 ng per $\mathrm{g}$ body weight), treated animals experienced a roughly $53 \%$ decrease in pro $\alpha 2$ (I) collagen mRNA accompanied by an up to $39 \%$ decrease in cytoskeletal actin mRNA (25). Another study conducted on rats reported a decreased amount of mature collagen-I and -III in the left ventricles of hypertrophic rats treated with $80 \mathrm{ng}$ T3 per g body weight (28). Both studies found a correlation between misregulated interstitial protein synthesis and the onset of cardiac hypertrophy $(25,28)$, which corroborates the recent finding that postnatal cardiac fibroblasts create a non-permissive environment for CM cytokinesis in murine hearts by ECM-mediated interactions (26). While these studies suggest that thyroid hormone further inhibits murine cardiac regeneration through regulation of the ECM (26), CM cytoskeletal (25), and fibrotic structural integrity (28), more research should verify these claims across phylogeny.

The role of thyroid hormone in heart regeneration via ECM regulation has gained support by a recent study in Xenopus laevis. Marshall et al. found that alterations 
in metamorphosis-driven rise in thyroid hormone levels coincides with misregulated ECM structure and expression levels more so than changes in CM proliferative capacity (29). X. laevis is an anuran amphibian recognized for its robust limb regenerative capacity (29) and is surprisingly not a common subject of cardiac regenerative studies. $X$. laevis undergoes a restriction in cardiac regeneration partially due to ECM gene misregulation, ECM protein accumulation at injury sites, and insignificant changes in $\mathrm{CM}$ proliferation (29). Similar to mammals, the cardiac regenerative potential of $X$. laevis larvae progressively diminishes in conjunction with a developmental postnatal rise in thyroid hormone (29). Increased thyroid hormone regulates the progression of metamorphosis and contributes to the loss of tadpoles' complete cardiac regenerative capacity observed in post-metamorphic juvenile and adult stages (29). Moreover, when treated with exogenous $0.1 \mathrm{nM}$ T3 or a thyroid hormone synthesis inhibitor sodium perchlorate before the cardiac injury, both juvenile and adult $X$. laevis displayed restricted cardiac regeneration characterized by transcriptional changes in ECM genes, an accumulation of ECM proteins, and a decrease in fibrotic resorption at the infarction site (29). Counter to Hirose et al.'s findings (2), these animals showed no significant changes in CM proliferation both locally at the injury site as well as globally, suggesting that diminished cardiac regeneration cannot be explained entirely by CM proliferation, but also involves thyroid hormone-dependent changes in ECM transcription and kinetics (29). However, in the zebrafish heart regeneration study by Hirose et al. (2), $5 \mathrm{nM}$ T3 was given to the tank water from the second-day post-cardiac injury to the end of experiments and an approximately $50 \%$ reduction of CM proliferation was observed. Thus, the different duration and dose of T3 used in the previous two studies likely account for the observed different effect on CM proliferation.

Evolutionary perspectives propose that elevations in circulating thyroid hormone levels across phylogeny and ontogeny coincided with the poikilotherm-toendotherm transition and the limited cardiac regenerative capacity of mammals likely originated as a tradeoff for the acquisition of endothermy (2). A recent study conducted on both mice and zebrafish found high levels of thyroid hormones promote CM polyploidy likely due to cytokinesis failure (2). Through a phylogenetic analysis of vertebrate CM ploidy, Hirose et al. uncovered that certain monotreme, edentate, cetacean, and chiropteran species have unusually high percentages of diploid CMs in the adult heart (2). Diploid CM abundance across 41 species conforms to Kleiber's law (2), the 3/4-power law scaling metabolism with bodyweight (30), and decreases when the standard metabolic rate, body temperature, and thyroid hormone levels increase during the ectothermto-endotherm transition (2). Furthermore, mammals displayed a linear relationship between standard body temperature and diploid CM percentage, supporting theories that the onset of endothermy correlates with diminished cardiac regenerative potential (2). Intriguingly, thyroid hormones have been identified as major regulators of energy metabolism (31). It is possible that elevated metabolic rates and the evolution of endothermy offered early mammals survival advantages as they inhabited a temporally wide nocturnal niche and expanded into regions of colder climates previously unexplored by reptiles (2). The loss of proliferation-competent cells and the decline of regenerative capacity in the heart and other organs were seemingly not under selection pressure in early mammalian evolution (2).

In both rodent and human hearts, there are significant increases of $\mathrm{CM}$ number and/or proliferation in the postnatal window $(13,15,16,19,22,32)$. This is the same period when thyroid hormone levels increase (33). Why can thyroid hormones not completely inhibit the significant increase in CM proliferation? First, the timing and dose are likely important. In rodents, circulating thyroid hormone levels rise after birth and peak around P14 (33). The concentration of thyroid hormones in the first several days might not be high enough to inhibit CM proliferation and thus, there are significant $\mathrm{CM}$ number increases during the first several days after birth, but not much afterwards (15). This hypothesis is supported by the study of Gerdes et al. which showed that daily $\mathrm{T} 4$ treatment of postnatal rat pups resulted in a 19\% decrease in the number of ventricular myocytes (17). Secondly, there are likely thyroid hormone-independent mechanisms. In P14 mutant mice with a deficiency in thyroid hormone signaling, only approximately $3 \%$ of CMs were in the cell cycle and only $30 \%$ of CMs were diploid, indicating that most CMs already withdrew from the cell cycle and became polyploid after cytokinesis failure (2). These findings suggest that there are additional major physiological drivers of postnatal CM cell-cycle exit waiting for discovery.

\section{Roles of vitamin $D$ in regulating cardiomyocyte proliferation and cardiac regeneration}

Vertebrates primarily obtain vitamin D via dietary means and chemical reactions on steroid precursors in the form

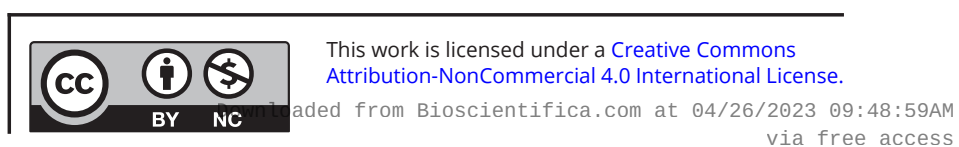


Table 1 A summary of reported effects of thyroid hormones, vitamin D, and glucocorticoids on cardiomyocyte proliferation and heart regeneration.

\begin{tabular}{|c|c|c|c|c|}
\hline \multirow[b]{2}{*}{ Hormone } & \multirow[b]{2}{*}{ Function } & \multicolumn{2}{|l|}{ Cardiomyocyte proliferation } & \multirow[b]{2}{*}{ Heart regeneration } \\
\hline & & In vitro & $\begin{array}{l}\text { In vivo (perinatal growth or adult injury } \\
\text { repair) }\end{array}$ & \\
\hline \multirow{3}{*}{ Thyroid hormones } & No effect & Mouse (2), chicken (12) & & \\
\hline & Inhibit & & $\begin{array}{l}\text { Zebrafish (2), rat (16), mouse (2), } \\
\text { sheep (10) }\end{array}$ & Zebrafish (2), frog (19), mouse (2) \\
\hline & Promote & & Mouse $(13,17)$, sheep $(11)$ & \\
\hline \multirow[t]{3}{*}{ Vitamin D } & No effect & & Mouse (36) & \\
\hline & Inhibit & Mouse $(36,42)$, rat $(41,43)$ & Mouse (36) & \\
\hline & Promote & Mouse $(40)$ & Zebrafish (40) & Zebrafish (40) \\
\hline \multirow[t]{2}{*}{ Glucocorticoid } & No effect & & Mouse (36) & \\
\hline & $\begin{array}{l}\text { Inhibit } \\
\text { Promote }\end{array}$ & Mouse (36), rat (51) & Zebrafish (46), mouse (36), rat (50) & Zebrafish (46) \\
\hline
\end{tabular}

of UV irradiation and sequential hydroxylations $(34,35)$. In the kidney, vitamin $\mathrm{D}$ is consecutively hydroxylated to produce ercalcitriol (vitamin D2) and the most active form $1 \alpha, 25$ dihydroxyvitamin $\mathrm{D}(1 \alpha, 25(\mathrm{OH}) 2 \mathrm{D})$ or calcitriol (vitamin D3) $(35,36,37)$. Vitamin D's cognate receptor is vitamin $\mathrm{D}$ receptor (VDR) a ligand-dependent member of the nuclear receptor superfamily $(35,38)$. Upon binding of vitamin $\mathrm{D}, \mathrm{VDR}$ regulates transcription and cellular pathways by binding to vitamin $\mathrm{D}$ response elements located in the effector regions of target genes, recruiting coregulators and cofactors, and undergoing homo and heterodimerization with itself and other receptors such as $\mathrm{TR}$, but most commonly retinoid $\mathrm{X}$ receptors (RXRs) $(34,38,39)$.

Vitamin D has been documented to exhibit differential effects on CM proliferation across phylogeny with mammals generally experiencing anti-proliferative effects (34) and lower vertebrates such as zebrafish undergoing increases in CM proliferation (40). Vitamin $\mathrm{D}$ analogs have been reported to exert anti-proliferative effects most commonly in mammalian cells (34) as well as mammalian CMs and CM-derived cell lines (41, 42, 43) by possibly diminishing the amount of various cyclins and/or upregulating cyclin-dependent kinase (CDK) inhibitors (34). In support, in vitro and in vivo treatments with VDR agonists resulted in a significant decrease in neonatal mouse CM proliferation (36). However, CM-specific VDR knockout mice revealed no significant change in CM proliferation at P14 as characterized by insignificant changes in mononuclear CM percentage as well as mitotic and cytokinetic CM activity compared to controls (36). These findings suggest that exogenous vitamin D is sufficient to inhibit CM proliferation, but the endogenous vitamin D signaling pathway is not necessary for neonatal CM cell-cycle exit in vivo (36). Intriguingly, vitamin $\mathrm{D}$ signaling has been documented in zebrafish as a mitogen for various cell types and an endogenous regulator of organ size (40). Adult zebrafish underwent partial ventricle resections and at 6-7 days post-resection were either administered the VDR agonist alfacalcidol or experienced heat shock treatment to inhibit VDR (40). The $10 \mathrm{nM}$ alfacalcidol condition produced a $41 \%$ increase in proliferating CMs, while VDR inhibition resulted in a $69 \%$ decrease in proliferating CMs, suggesting VDR signaling is both sufficient and necessary for zebrafish CM proliferation and cardiac regeneration (40). Disparities in the $\mathrm{CM}$ proliferative responses between mice and zebrafish imply that the effects of vitamin D signaling on cardiac regeneration are highly species and contextdependent $(36,40)$.

Han et al. demonstrated that supraphysiological vitamin $\mathrm{D}$ expression in neonatal and adult zebrafish promotes cardiac regeneration (40). Heightened levels of vitamin D correlated with significant increases in CM proliferation primarily through increased mitogenic cell cycle activity in $G_{1}$ as well as the regulation of organ size and growth (40). Similarly, alterations in cardiacspecific VDR signaling sufficiently regulated mouse CM proliferation and heart size in vitro (40). In contrast to Cutie et al. (36), treatment of neonatal mouse CMs with $1 \mu \mathrm{M}$ alfacalcidol increased proliferating CM frequency in a dose-dependent manner (40). The group proposed that vitamin D supplementation is sufficient to induce cell cycle progression in mouse and zebrafish CMs (40). The reason for the divergence in neonatal mouse CM proliferation results between Cutie et al. and Han et al. is unknown. Overall in mammals, literature has demonstrated exogenous vitamin $\mathrm{D}$ to exhibit inhibitory effects on CM proliferation by repressing CM cell cycling (36). The major differences in the effects of VDR signaling on CM proliferation between lower vertebrates and mammals are most likely due to the variability in vitamin D context,

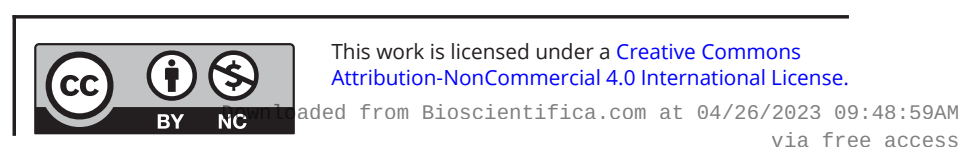


dosage, and species-dependent effects on VDR canonical and non-canonical regulatory pathways $(36,40)$.

Across phylogeny, vitamin $\mathrm{D}$ deficiencies are a common predictor of cardiac disease and mortality for which the administration of active vitamin D exhibits cardioprotective effects, while exceeding normal physiological levels has been shown to contribute to cardiac hypertrophy and impaired heart function (44, 45). Human heart failure patients with deficiencies in circulating calcitriol levels displayed significantly higher levels of ventricular cardiac fibrosis and myocardial interstitial collagen than controls, implying that vitamin D plays an important role in mammalian heart failure recovery specifically in regards to adult non-CMs (44). Hypovitaminosis D in humans is a common predictor of cardiac disease and mortality (45). Patients experiencing left ventricular hypertrophy (LVH), a prominent risk factor for cardiac disease, tend to exhibit decreased serum levels of vitamin D (45). Upon treatment with calcitriol, patients with LVH experienced a cardioprotective decrease in cardiac hypertrophy (45). CM-specific deletions of VDR and global VDR knockouts in mice both resulted in CM hypertrophy and overall heart enlargement, suggesting that VDR plays an important role in preventing cardiac hypertrophy and preserving normal cardiac function in mice as well (35). Similarly in zebrafish, DN VDR mutants sustained prominent scarring at 50 days post-ventricularresection, implicating VDR as an essential regulator of fibrotic resorption and cardiac regeneration (40). DN-VDR zebrafish also exhibited a $27 \%$ reduction in their heartto-body size ratio, showing cardiac VDR partly regulates heart size (40). Vitamin D's frequent interaction with an array of coregulators and other signaling molecules makes its effects on cardiac performance highly variable and context-dependent (45). Further studies should investigate the nuances in vitamin D dosage and its range of coregulators to better understand vitamin D's dose and context-dependent transition from a cardioprotector to a propogator of cardiac damage across species.

\section{Roles of glucocorticoids in regulating cardiomyocyte proliferation and cardiac regeneration}

Upon exposure to external stressors, vertebrates synthesize and secrete glucocorticoids from their anterior pituitary gland, which bind tissue-specific isoforms of their cognate receptor, the glucocorticoid receptor (GR), and activate cell-specific stress-response pathways (46). The primary forms of glucocorticoids in humans and rodents are cortisol and corticosterone, respectively (47). Intracellular cortisol levels are primarily controlled by $11 \beta$-Hydroxysteroiddehydrogenase (11ß-HSD) activity, which interconverts cortisol and its less active form, cortisone (47). Glucocorticoids canonically regulate the transcription of target cells through the binding of GR, a ligand-dependent transcription factor in the nuclear receptor superfamily $(47,48)$. When bound to glucocorticoids, GR regulates transcription through glucocorticoid response elements (GREs) mainly located in intragenic and intergenic positions relative to the promoters of target genes (48). Throughout its formation, GR progressively undergoes alternative splicing, translational variation, and posttranslational modifications, which produce a variety of GR isoforms with nuanced functionalities (47). The main GR isoforms in vertebrates are GR $\alpha$ and GR $\beta$ where GR $\alpha$ is predominantly found in most tissues including the heart (47).

Studies simulating exogenous glucocorticoid administration and GR loss of function across various species report a general decrease in CM proliferation and structural integrity, suggesting glucocorticoids sufficiently impair CM regenerative and proliferative growth (46), and are also necessary for normal heart development (49). GR's activation and subsequent epigenetic repression of Cyclin D2 genes has been reported to inhibit neonatal rat $\mathrm{CM}$ proliferation, promote neonatal rat CM hypertrophy, and increase CM binucleation $(50,51,52)$. Recently, administration of GR agonists was shown to produce a significant decrease in neonatal mouse CM proliferation in vitro and in vivo (36). Interestingly, P14 mice with CM-specific GR knockouts showed no significant change in CM proliferation (36), which implicates that glucocorticoids are unnecessary for neonatal mouse CM cell cycle withdrawal in vivo. Another study generated global GR knockout mice and discovered resulting irregular CM myofibril organization and $\alpha$-myosin heavy chain expression, implicating that GR deficiencies weaken CM structure (53). Further analyses should be conducted to determine if the predominant effects of GR deficiencies on cardiac regeneration stem from $\mathrm{CMs}$ or non-CM cells. In zebrafish, over ten-fold heightened levels of glucocorticoids prompted by crowding conditions impaired both cardiac regeneration post-cryoinjury and overall hyperplastic cardiac growth, despite zebrafish normally retaining robust cardiac regenerative potential well into adulthood (46).

Zebrafish subjected to myocardial cryoinjury and juvenile zebrafish with no prior cardiac injury both

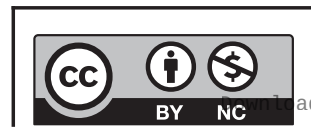


experienced a global suppression of $\mathrm{CM}$ proliferation following acute daily exposures to crowding (46). In regards to non-CMs, exogenous glucocorticoid administration in rats in the form of dexamethasone increased the amount of left ventricular mature collagens-I and -III (28). The proposed hypertrophic role of glucocorticoids was further supported by a study that found in mice, glucocorticoids induce cardiac fibrosis and inflammation (54). Although studies suggest glucocorticoids are essential for cardiac development (53), more studies should aim to differentiate the prominent GR isoforms and mechanisms that contribute to glucocorticoid-mediated cardiac impairments and hypertrophy following exogenous glucocorticoid administration (46).

In addition to interfering with $\mathrm{CM}$ proliferation, exogenous glucocorticoids interfere with overall cardiac regeneration and function across phylogeny $(46,49)$. In a study by Sallin et al., zebrafish were exposed to perceived acute stressors for 30 days post-ventricular cryoinjury (dpci) through crowding (46). Cardiac regenerative capacity was greatly diminished with only $33 \%$ of the affected zebrafish replacing transient fibrosis and the majority experiencing a global suppression of CM proliferation (46). Furthermore, daily crowding during the initial 1-14 dpci period inhibited cardiac regeneration in $78 \%$ of subjects, while the following $15-30$ dpci proved insignificant, suggesting the initial 14-day period plays a critical role in establishing effective cardiac regeneration (46). Surprisingly, whole transcriptome analyses at 14 dpci when zebrafish undergo a transition from scar formation to resorption revealed that significantly downregulated genes primarily reside in the endocardium surrounding affected CMs, indicating a partly non-cellautonomous cardiac regenerative mechanism in zebrafish (46). Similarly, prenatal mice with GR global knockouts underwent altered heart maturation with weakened CM structure, lowered levels of $\alpha$-myosin heavy chain protein, and decreased calcium handling proteins (53). Mice with a CM-specific GR deletion frequently died prematurely at a median life span of approximately 7 months due to the development of dilated cardiomyopathy and related cardiac pathologies (47). While exogenous glucocorticoids significantly diminish cardiac regenerative potential, GR loss of function studies also demonstrates glucocorticoids are necessary for normal cardiac development.

Glucocorticoids have also been shown to regulate cardiac function through the binding of mineralocorticoid receptor (MR), which is another nuclear receptor with a similar structure to GR and shares many of the same target genes, but has largely antagonistic effects on heart contractile functions $(47,55)$. MR has a modular structure similar to GR composed of a DNA-binding domain (DBD), ligand-binding domain (LBD), and a $\mathrm{N}$-terminal transactivation domain (NTD) (47). MR's DBD is approximately $94 \%$ similar to that of GR, allowing for MR to bind many of the same DNA response elements as GR, including GREs, while exerting antagonistic transcriptional regulatory effects $(47,55)$. In the previously stated study that found that prenatal mice with CM-specific GR knockouts die prematurely due to the development of various cardiac pathologies (47), an interesting development occurred where double knockout mouse models with inactivated MR in addition to GR in CMs did not die prematurely and exhibited normal cardiac morphology (47). The rescue of normal cardiac morphology in double knockout mice suggests that MR exacerbates and progresses cardiac disease when experiencing myocardial stress (55). Future studies on the effects of glucocorticoid signaling on $\mathrm{CM}$ and cardiac regeneration should also investigate the role of MR signaling, which appears closely related and antagonistic to GR signaling (55). Concurrent modulation of both GR and MR could produce novel therapies to treat cardiac damage.

\section{Discussion}

The effects of thyroid hormone, vitamin D, and glucocorticoid signaling on CM proliferation and cardiac regeneration are found to vary in age, context, species, and dose-dependent manner (Fig. 1). Their roles in cardiac development and disease progression facilitated by their respective canonical signaling pathways through nuclear receptors are further complicated by extensive downstream signaling pathways $(16,46)$, an array of coregulators and cofactors $(34,38)$, and transcriptional response element availability (48). Most studies focus on correlations and causal relationships between endocrine signaling pathways and their effects on cardiac regeneration, but few expound on their findings' significance in macroscopic evolutionary developments. Comprehensive phylogenetic studies provide an effective means for understanding crossspecies divergence in cardiac regeneration (4), which can then be applied to humans in hopes of realizing cardiac therapies. The theory that the phylogenetic divergence in cardiac regenerative capacity relates to the evolutionary transition from ectothermy to endothermy provides a statistically based evolutionary relationship with thyroid

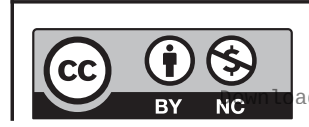

This work is licensed under a Creative Commons Attribution-NonCommercial 4.0 International License. ded from Bioscientifica.com at 04/26/2023 09:48:59AM 
hormone signaling as one of the main proponents (2). Evolutionary analysis should also be pursued for vitamin D and glucocorticoids, which may not surface as strongly supported relationships, but whose insights would extend far beyond cardiac function and regenerative potential.

Now that a range of indicators for cardiac regenerative capacity have been identified and their varying effects documented, it is of pressing interest to explore their combinatorial effects with an intent to optimize cardiac regenerative responses. In order to account for the possibility that thyroid hormone signaling modifies the effects of a CM-specific GR or VDR deletion, Cutie et al. bred GR and VDR CM-specific deletions independently and in conjunction with TR, but neither combined loss of function genotype demonstrated a significant change in $\mathrm{CM}$ proliferation relative to controls (36). The results suggest that the individual effects of each hormone on cardiac regeneration are largely influenced by their context and require more in-depth studies into their nuanced combinatorial effects $(36,56)$. While most research on cardiac regeneration focuses on CM-specific regenerative mechanisms, growing research has highlighted the importance of accounting for non-cell-autonomous regenerative mechanisms with a focus on interstitial proteins such as cardiac fibroblasts $(26,28)$. Aside from endocrine signaling pathways, other modifications that significantly altered cardiac regeneration and could be studied in combination with endocrine signaling include: ECM proteins and structure (26), alterations in local nonCMs such as cardiac fibroblasts $(26,28)$, hormone-specific transcriptional response elements that interact with target gene effectors $(7,39,48)$, and the modulation of target gene transcription by coregulator recruitment and local chromatin assembly (38). In all, each of the three hormones exert both cardioprotective and damaging effects. By accounting for the temporal and contextdependent confounding factors in cardiac regenerative responses across phylogeny, the cardioprotective effects of thyroid hormone, vitamin D, and glucocorticoid could be exploited as revolutionary therapies for heart failure.

\section{Declaration of interest}

The authors declare that there is no conflict of interest that could be perceived as prejudicing the impartiality of this review.

\section{Funding}

This research was made possible by funding from the University of California, Berkeley Undergraduate Research Apprenticeship Program's Summer Fellowship (A A), NIH F31 Fellowship (S C), NIH (R01HL138456),
Department of Defense (W81XWH1910206), Program for Breakthrough Biomedical Research, and UCSF Eli and Edythe Broad Center of Regeneration Medicine and Stem Cell Research Seed Grant (G N H).

\section{Acknowledgements}

We thank the members of the Huang Lab for their continuous support and contributions throughout the entire writing process.

\section{References}

1 Nowbar AN, Gitto M, Howard JP, Francis DP \& Al-Lamee R Mortality from Ischemic heart disease. Circulation: Cardiovascular Quality and Outcomes 201912 e005375. (https://doi.org/10.1161/ CIRCOUTCOMES.118.005375)

2 Hirose K, Payumo AY, Cutie S, Hoang A, Zhang H, Guyot R, Lunn D, Bigley RB, Yu H, Wang J, et al. Evidence for hormonal control of heart regenerative capacity during endothermy acquisition. Science 2019364 184-188. (https://doi.org/10.1126/science.aar2038)

3 Senyo SE, Steinhauser ML, Pizzimenti CL, Yang VK, Cai L, Wang M, Wu TD, Guerquin-Kern JL, Lechene CP \& Lee RT. Mammalian heart renewal by pre-existing cardiomyocytes. Nature 2013493 433-436. (https://doi.org/10.1038/nature11682)

4 Vivien CJ, Hudson JE \& Porrello ER. Evolution, comparative biology and ontogeny of vertebrate heart regeneration. NPJ Regenerative Medicine 20161 16012. (https://doi.org/10.1038/ npjregenmed.2016.12)

5 Porrello ER, Mahmoud AI, Simpson E, Hill JA, Richardson JA, Olson EN \& Sadek HA. Transient regenerative potential of the neonatal mouse heart. Science 2011331 1078-1080. (https://doi. org/10.1126/science.1200708)

6 González-Rosa JM, Burns CE \& Burns CG. Zebrafish heart regeneration: 15 years of discoveries. Regeneration 20174 105-123. (https://doi.org/10.1002/reg2.83)

7 Grais IM \& Sowers JR. Thyroid and the heart. American Journal of Medicine 2014127 691-698. (https://doi.org/10.1016/j. amjmed.2014.03.009)

8 Davis PJ, Goglia F \& Leonard JL. Nongenomic actions of thyroid hormone. Nature Reviews: Endocrinology 201612 111-121. (https:// doi.org/10.1038/nrendo.2015.205)

9 Hönes G, Helena R, Logan J, Liao X, Werbenko E, Pollard A, Praestholm S, Siersbaek M, Rijntjes E, Gassen J, et al. Noncanonical thyroid hormone signaling mediates cardiometabolic effects in vivo. PNAS 2017114 E11323-E11332. (https://doi.org/10.1073/ pnas.1706801115)

10 Chattergoon NN, Giraud GD \& Thornburg KL. Thyroid hormone inhibits proliferation of fetal cardiac myocytes in vitro. Journal of Endocrinology 2007192 R1-R8. (https://doi.org/10.1677/JOE-06-0114)

11 Segar JL, Volk KA, Lipman MH \& Scholz TD. Thyroid hormone is required for growth adaptation to pressure load in the ovine fetal heart. Experimental Physiology 201398 722-733. (https://doi. org/10.1113/expphysiol.2012.069435)

12 Svensson Holm AC, Lindgren I, Osterman H \& Altimiras J. Thyroid hormone does not induce maturation of embryonic chicken cardiomyocytes in vitro. Physiological Reports 20142 e12182. (https:// doi.org/10.14814/phy2.12182)

13 Naqvi N, Li M, Calvert JW, Tejada T, Lambert JP, Wu J, Kesteven SH Holman SR, Matsuda T, Lovelock JD, et al. A proliferative burst during preadolescence establishes the final cardiomyocyte number. Cell 2014157 795-807. (https://doi.org/10.1016/j.cell.2014.03.035)

14 Soonpaa MH, Zebrowski DC, Platt C, Rosenzweig A, Engel FB \& Field LJ. Cardiomyocyte cell-cycle activity during preadolescence. Cell 2015163 781-782. (https://doi.org/10.1016/j.cell.2015.10.037) https://ec.bioscientifica.com

https://doi.org/10.1530/EC-20-0503 (c) 2021 The authors Published by Bioscientifica Ltd
This work is licensed under a Creative Commons Attribution-NonCommercial 4.0 International License. ded from Bioscientifica.com at 04/26/2023 09:48:59AM 
15 Alkass K, Panula J, Westman M, Wu TD, Guerquin-Kern JL \& Bergmann O. No evidence for cardiomyocyte number expansion in preadolescent mice. Cell 2015163 1026-1036. (https://doi. org/10.1016/j.cell.2015.10.035)

16 Tan L, Bogush N, Naib H, Perry J, Calvert JW, Martin DIK, Graham RM, Naqvi N \& Husain A. Redox activation of JNK2 $\alpha 2$ mediates thyroid hormone-stimulated proliferation of neonatal murine cardiomyocytes. Scientific Reports 20199 17731. (https://doi. org/10.1038/s41598-019-53705-1)

17 Gerdes AM, Kriseman J \& Bisop SP. Changes in myocardial cell size and number during the development and reversal of hyperthyroidism in neonatal rats. Laboratory Investigation $1983 \mathbf{4 8}$ 598-602.

18 Burgi U, Burgi-Saville ME, Ziegler F, Krahenbuhl L, Huber G \& Schoch C. Food intake, body and heart composition, and heart rate in T3 plus atenolol-treated rats. American Journal of Physiology 1989 256 E459-E466. (https://doi.org/10.1152/ajpendo.1989.256.4.E459)

19 Bergmann O, Zdunek S, Felker A, Salehpour M, Alkass K, Bernard S, Sjostrom SL, Szewczykowska M, Jackowska T, Remedios C, et al. Dynamics of cell generation and turnover in the human heart. Cell 2015161 1566-1575. (https://doi.org/10.1016/j.cell.2015.05.026)

20 Bergmann O, Zdunek S, Alkass K, Druid H, Bernard S \& Frisén J. Identification of cardiomyocyte nuclei and assessment of ploidy for the analysis of cell turnover. Experimental Cell Research $20113 \mathbf{3 1 7}$ 188-194. (https://doi.org/10.1016/j.yexcr.2010.08.017)

21 Srsen V, Fant X, Heald R, Rabouille C \& Merdes A. Centrosome proteins form an insoluble perinuclear matrix during muscle cell differentiation. BMC Cell Biology 200910 28. (https://doi. org/10.1186/1471-2121-10-28)

22 Mollova M, Bersell K, Walsh S, Savla J, Das LT, Park SY, Silberstein LE, Dos Remedios CG, Graham D, Colan S, et al. Cardiomyocyte proliferation contributes to heart growth in young humans. PNAS 2013110 1446-1451. (https://doi.org/10.1073/pnas.1214608110)

23 Lee YK, Ng KM, Chan YC, Lai WH, Au KW, Ho CY, Wong LY, Lau CP, Tse HF \& Siu CW. Triiodothyronine promotes cardiac differentiation and maturation of embryonic stem cells via the classical genomic pathway. Molecular Endocrinology 201024 1728-1736. (https://doi. org/10.1210/me.2010-0032)

24 Yang X, Rodriguez M, Pabon L, Fischer KA, Reinecke H, Regnier M, Sniadecki NJ, Ruohola-Baker H \& Murry CE. Tri-iodo-1-thyronine promotes the maturation of human cardiomyocytes-derived from induced pluripotent stem cells. Journal of Molecular and Cellular Cardiology 201472 296-304. (https://doi.org/10.1016/j. yjmcc.2014.04.005)

25 Yao J \& Eghbali M. Decreased collagen gene expression and absence of fibrosis in thyroid hormone-induced myocardial hypertrophy. Response of cardiac fibroblasts to thyroid hormone in vitro. Circulation Research 199271 831-839. (https://doi.org/10.1161/01. res.71.4.831)

26 Wu CC, Jeratsch S, Graumann J \& Stainier DYR. Modulation of mammalian cardiomyocyte cytokinesis by the extracellular matrix. Circulation Research 2020127 896-907. (https://doi.org/10.1161/ CIRCRESAHA.119.316303)

27 Li M, Parker BL, Pearson E, Hunter B, Cao J, Koay YC, Gunerantne O, James DE, Yang J, Lal S, et al. Core functional nodes and sex-specific pathways in human ischaemic and dilated cardiomyopathy. Nature Communications 202011 2843. (https://doi.org/10.1038/s41467-02016584-z)

28 Roy SG, Mishra S, Ghosh G \& Bandyopadhyay A. Thyroid hormone induces myocardial matrix degradation by activating matrix metalloproteinase-1. Matrix Biology 200726 269-279. (https://doi. org/10.1016/j.matbio.2006.12.005)

29 Marshall LN, Vivien CJ, Girardot F, Péricard L, Scerbo P, Palmier K, Demeneix BA \& Coen L. Stage-dependent cardiac regeneration in Xenopus is regulated by thyroid hormone availability. PNAS 2019 116 3614-3623. (https://doi.org/10.1073/pnas.1803794116)
30 Kleiber M. Body size and metabolism. Hilgardia 19326 315-353. (https://doi.org/10.3733/hilg.v06n11p315)

31 Mullur R, Liu YY \& Brent GA. Thyroid hormone regulation of metabolism. Physiological Reviews 201494 355-382. (https://doi. org/10.1152/physrev.00030.2013)

32 Li F, Wang X, Capasso JM \& Gerdes AM. Rapid transition of cardiac myocytes from hyperplasia to hypertrophy during postnatal development. Journal of Molecular and Cellular Cardiology 199628 1737-1746. (https://doi.org/10.1006/jmcc.1996.0163)

33 Li M, Iismaa SE, Naqvi N, Nicks A, Husain A \& Graham RM. Thyroid hormone action in postnatal heart development. Stem Cell Research 201413 582-591. (https://doi.org/10.1016/j.scr.2014.07.001)

34 Samuel S \& Sitrin MD. Vitamin D's role in cell proliferation and differentiation. Nutrition Reviews 200866 (Supplement 2) S116-S124. (https://doi.org/10.1111/j.1753-4887.2008.00094.x)

35 Chen S, Law CS, Grigsby CL, Olsen K, Hong TT, Zhang Y, Yeghiazarians Y \& Gardner DG. Cardiomyocyte-specific deletion of the vitamin D receptor gene results in cardiac hypertrophy. Circulation 2011124 1838-1847. (https://doi.org/10.1161/ CIRCULATIONAHA.111.032680)

36 Cutie S, Payumo AY, Lunn D \& Huang GN. In vitro and in vivo roles of glucocorticoid and vitamin D receptors in the control of neonatal cardiomyocyte proliferative potential. Journal of Molecular and Cellular Cardiology 2020142 126-134. (https://doi.org/10.1016/j. yjmcc.2020.04.013)

37 Bikle DD. Vitamin D metabolism, mechanism of action, and clinical applications. Chemistry and Biology 201421 319-329. (https://doi. org/10.1016/j.chembiol.2013.12.016)

38 Carlberg C \& Seuter S. A genomic perspective on vitamin D signaling. Anticancer Research 200929 3485-3493.

39 Hanel A, Malmberg HR \& Carlberg C. Genome-wide effects of chromatin on vitamin D signaling. Journal of Molecular Endocrinology 202064 R45-R56. (https://doi.org/10.1530/JME-19-0246)

40 Han Y, Chen A, Umansky KB, Oonk KA, Choi WY, Dickson AL, Ou J, Cigliola V, Yifa O, Cao J, et al. Vitamin D stimulates cardiomyocyte proliferation and controls organ size and regeneration in zebrafish. Developmental Cell 201948 853.e5-863.e5. (https://doi.org/10.1016/j. devcel.2019.01.001)

41 Hlaing SM, Garcia LA, Contreras JR, Norris KC, Ferrini MG \& Artaza JN. 1,25-Vitamin D3 promotes cardiac differentiation through modulation of the Wnt signaling pathway. Journal of Molecular Endocrinology 201453 303-317. (https://doi.org/10.1530/JME-140168)

42 Nibbelink KA, Tishkoff DX, Hershey SD, Rahman A \& Simpson RU. $(\mathrm{OH})$-vitamin D3 actions on cell proliferation, size, gene expression, and receptor localization, in the HL-1 cardiac myocyte. Journal of Steroid Biochemistry and Molecular Biology 2007103 533-537. (https:// doi.org/10.1016/j.jsbmb.2006.12.099)

43 O'Connell TD, Berry JE, Jarvis AK, Somerman MJ \& Simpson RU. 1,25-Dihydroxyvitamin D3 regulation of cardiac myocyte proliferation and hypertrophy. American Journal of Physiology 1997272 H1751-H1758. (https://doi.org/10.1152/ ajpheart.1997.272.4.H1751)

44 Meredith A, Boroomand S, Carthy J, Luo Z \& Mcmanus B. 1,25 Dihydroxyvitamin D3 inhibits TGF 1 1-mediated primary human cardiac myofibroblast activation. PLOS ONE 201510 e0128655. (https://doi.org/10.1371/journal.pone.0128655)

45 Artaza JN, Mehrotra R \& Norris KC. Vitamin D and the cardiovascular system. Clinical Journal of the American Society of Nephrology 20094 1515-1522. (https://doi.org/10.2215/CJN.02260409)

46 Sallin P \& Jaźwińska A. Acute stress is detrimental to heart regeneration in zebrafish. Open Biology 20166 160012. (https://doi. org/10.1098/rsob.160012)

47 Cruz-Topete D, Oakley RH \& Cidlowski JA. Glucocorticoid signaling and the aging heart. Frontiers in Endocrinology 202011 347. (https:// doi.org/10.3389/fendo.2020.00347)

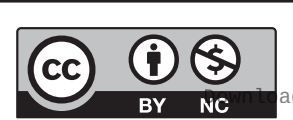

This work is licensed under a Creative Commons Attribution-NonCommercial 4.0 International License. ded from Bioscientifica.com at 04/26/2023 09:48:59AM 
48 Oakley RH \& Cidlowski JA. The biology of the glucocorticoid receptor: new signaling mechanisms in health and disease. Journal of Allergy and Clinical Immunology 2013132 1033-1044. (https://doi. org/10.1016/j.jaci.2013.09.007)

49 Oakley RH \& Cidlowski JA. Glucocorticoid signaling in the heart: a cardiomyocyte perspective. Journal of Steroid Biochemistry and Molecular Biology 2015153 27-34. (https://doi.org/10.1016/j. jsbmb.2015.03.009)

50 Gay MS, Li Y, Xiong F, Lin T \& Zhang L. Dexamethasone treatment of newborn rats decreases cardiomyocyte endowment in the developing heart through epigenetic modifications. PLoS ONE 2015 10 e0125033. (https://doi.org/10.1371/journal.pone.0125033)

51 Gay MS, Dasgupta C, Li Y, Kanna A \& Zhang L. Dexamethasone induces cardiomyocyte terminal differentiation via epigenetic repression of cyclin D2 gene. Journal of Pharmacology and Experimental Therapeutics 2016358 190-198. (https://doi.org/10.1124/jpet.116.234104)

52 Whitehurst RM, Zhang M, Bhattacharjee A \& Li M. Dexamethasone induced hypertrophy in rat neonatal cardiac myocytes involves an elevated L-type Ca2+ current. Journal of Molecular and Cellular Cardiology 199931 1551-1558. (https://doi.org/10.1006/jmcc.1999.0990)
53 Rog-Zielinska EA, Thomson A, Kenyon CJ, Brownstein DG, Moran CM, Szumska D, Michailidou Z, Richardson J, Owen E, Watt A, et al. Glucocorticoid receptor is required for foetal heart maturation. Human Molecular Genetics 201322 3269-3282. (https:// doi.org/10.1093/hmg/ddt182)

54 Yang M, Zheng J, Miao Y, Wang Y, Cui W, Guo J, Qiu S, Han Y, Jia L, Li H, et al. Serum-glucocorticoid regulated kinase 1 regulates alternatively activated macrophage polarization contributing to angiotensin II-induced inflammation and cardiac fibrosis. Arteriosclerosis, Thrombosis, and Vascular Biology 201232 1675-1686. (https://doi.org/10.1161/ATVBAHA.112.248732)

55 Oakley RH, Cruz-Topete D, He B, Foley JF, Myers PH, Xu X, GomezSanchez CE, Chambon P, Williz MS \& Cidlowski JA. Cardiomyocyte glucocorticoid and mineralocorticoid receptors directly and antagonistically regulate heart disease in mice. Science Signaling 2019 12 eaau9685. (https://doi.org/10.1126/scisignal.aau9685)

56 Easterling MR, Engbrecht KM \& Crespi EJ. Endocrine regulation of regeneration: linking global signals to local processes. General and Comparative Endocrinology 2019283 113220. (https://doi. org/10.1016/j.ygcen.2019.113220)

Received in final form 16 November 2020

Accepted 14 December 2020

Accepted Manuscript published online 15 December 2020
This work is licensed under a Creative Commons Attribution-NonCommercial 4.0 International License. ded from Bioscientifica.com at 04/26/2023 09:48:59AM via free access 